\title{
Common Proper Motion Search for Faint Companions Around Early-Type Field Stars - Progress Report
}

\author{
Valentin D. Ivanov, G. Chauvin, C. Foellmi, M. Hartung, N. \\ Huélamo, C. Melo, D. Nürnberger and M. Sterzik \\ European Southern Observatory, Ave. Alonso de Cordova 310\%, Casilla 19, \\ Santiago 19001, Chile
}

September 30, 2005

\begin{abstract}
The multiplicity of early-type stars is still not well established. The derived binary fraction is different for individual star forming regions, suggesting a connection with the age and the environment conditions. The few studies that have investigated this connection do not provide conclusive results. To fill in this gap, we started the first detailed adaptive-optic-assisted imaging survey of earlytype field stars to derive their multiplicity in a homogeneous way. The sample has been extracted from the Hipparcos Catalog and consists of 341 BA-type stars within $\sim 300$ pc from the Sun. We report the current status of the survey and describe a Monte-Carlo simulation that estimates the completeness of our companion detection.
\end{abstract}

Keywords: stars:binaries:general, stars:binaries:visual, stars:early-type

\section{Introduction}

The multiplicity of pre-main sequence (PMS) and main sequence (MS) late-type stars have been extensively studied: Duquennoy \& Mayor (1991), Reipurth \& Zinnecker (1993), Prosser et al. (1994), Brandner et al. (1996). PMS late-type stars in low-density clouds like Taurus show higher binary fractions than PMS late-type stars formed in massive and dense star forming regions (SFR) like Orion. This difference was explained with an environmental dependence of the binary fraction: low-mass stars born in dense SFRs show a higher probability of dynamic interactions with more massive members, so that they are ejected resulting in a low binary fraction, as shown by Sterzik \& Durisen (1995). On the other hand, stars in low-density SFRs undergo low rate of stellar encounters, resulting in a higher binary fraction.

It is unclear if this result remains valid for early-type stars, mostly because of the difficult detection of faint companions near bright $\mathrm{O}-$ and B-type stars. The works of Petr et al. (1998), Preibisch et al. (1999) and Shatsky \& Tokovinin (2002) indicate that the binary fraction of earlytype stars varies from one SFR to another but current statistical basis is still not solid enough. The age of the SFRs can also affect the binary statistics: older systems are expected to have undergone through more

(C) 2020 Kluwer Academic Publishers. Printed in the Netherlands. 
dynamical interaction, reducing their binarity fraction in comparison with the younger ones, as point by Tokovinin et al. (1999).

\section{The Survey}

The cluster multiplicity studies can cover only a limited range of density and age. This prompted us to estimate the binarity fraction of a representative, volume-limited sample of early-type field stars. We designed a survey able to detect at $\sim 10 \sigma$ level an M4-type companion at the mean distance of our sample $(\sim 200 \mathrm{pc})$ down to 0.4 arcsec separation from $100 \mathrm{Myr}$ old A-type primary. The companions around B-type stars will be younger ( $\sim 10 \mathrm{Myr})$, brighter, and easier to detect. Last but not least, the physical nature of the candidate components is verified by their common proper motion. Our goal is to compare the properties such as incidence and mass ratio of the multiple stars in the field and in different star-forming regions.

\section{Sample Selection}

The sample stars were selected according to the following criteria:

- Apparent color $B-V \leq 0.2 \mathrm{mag}$ - conservative criterion met by all unreddened BA stars, and a few contaminating later-type stars. The spectral types for all stars were verified to be BA.

- The sample contains field stars only. Members of the OB-associations listed in de Zeeuw et al. (1999) were excluded.

- Distance $\mathrm{D} \leq 300 \mathrm{pc}$ from the Sun (HIPPARCOS). At $\mathrm{D}=300 \mathrm{pc}$ the telescope's diffraction limit of 0.07 arcsec corresponds to $21 \mathrm{~A} . \mathrm{U}$ (Shatsky \& Tokovinin (2002) probed separations 45-900 A.U).

- Proper motions $\geq 27$ mas/yr allowing to confirm/reject physical companion candidates taking two epoch separated by 1-2 yr.

- The apparent $V=5-6 \mathrm{mag}$, so the targets are suitable self-references for NACO even under poor weather conditions.

- The stars have DEC $\leq 0$ deg, i.e. visible from the VLT.

The final sample contains 341 field B- and A-type stars. The average distance is $114 \mathrm{pc}$, the median distance is $104 \mathrm{pc}$.

\section{Observations and Current Status}

The observations were carried out with NAOS-CONICA (Nasmyth Adaptive Optics System - Near-Infrared Imager and Spectrograph) at 
the ESO VLT over the last two years. The pixel scale was 27.03 mas px $^{-1}$, giving $27.7 \times 27.7$ arcsec field of view. Each target was observed at 9 different position on the detector, collecting total of $\sim 7.5 \mathrm{~min}$ of integration. The data reduction includes sky subtraction, flat-fielding, aligning and combination of the images into a single frame. Next, we perform PSF fitting/subtraction and search for faint companions.

As of Sept 2005 we have observed 196 objects from our sample. We have analyzed 152 of them: 81 appear single, and 71 show companion candidates whose nature will be tested with the second epoch observations with $\geq 2$ yr baseline.

\section{Analysis: Modeling the Survey}

To estimate the sensitivity and the completeness of the survey we have created a Monte-Carlo simulation that takes into account all available information for the survey stars (Fig. 1). The model input parameters are:

- the known distances, spectral types, absolute luminosities for all primaries;

- adopted binarity fraction;

- secondary star mass randomly sampled from the Kroupa et al. (1993) IMF;

- secondary star's spectral type and absolute magnitude calculated from the mass;

- orbital periods - randomly generated from Duquennoy \& Mayor (1991) distribution;

- major axis calculated from the Kepler's low and the period;

- random ellipticity, random orbital inclination;

- visibility criterion based on the magnitude difference and the angular separation between the primary and the companion.

The model predicts: the distributions of periods, angular separations, magnitude differences and spectral types for the detected binaries. The simulations indicate that we will detect about $2 / 3$ of the physical companions.

\section{Acknowledgements}

We are grateful to our colleagues from the ESO-Paranal Science Operations Department who carried out these observation in Service Mode. 

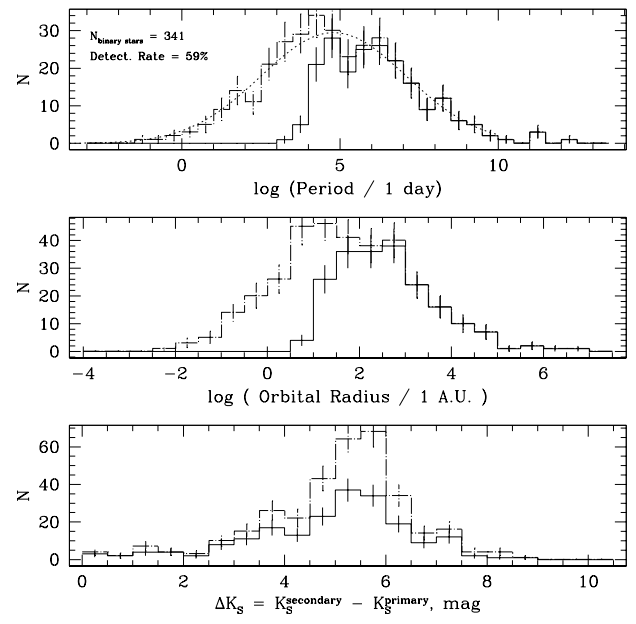

Figure 1. Monte-Carlo simulation of the survey completeness: orbital period distribution (top panel), orbital radii distribution (middle), and primary-secondary $K$-band magnitude difference (bottom). The dot-dashed lines show the "true" adopted/generated distributions and the solid lines are the "observed" distributions after the observational detectability conditions have been applied. The theoretical period distribution of Duquennoy \& Mayor (1991) is also shown (doted line on the top panel).

\section{References}

Brandner, W., Alcala, J.M., Moneti, A., \& Zinnecker, H. 1996, A\&A, 307, 121

de Zeeuw, P.T., Hoogerwerf, R., de Bruijne, J.H.J., Brown, A.G.A., \& Blaauw, A. 1999, AJ, 117, 354

Duquennoy, A. \& Mayor, M. 1991, A\&A, 248, 485

Kroupa, P., Tout, C.A. \& Gilmore, G. 1993, MNRAS, 262, 545

Petr, M.G., Coude Du Foresto, V., Beckwith, S.V.W., Richichi, A., McCaughren, M.J. 1998, ApJ, 500, 825

Preibisch, T., Balega, Y., Hofmann, K.-H., Weiglet, G., \& Zinnecker, H. 1999, New Astr., 4, 531

Prosser, C.F., Stauffer, J.R., Hartmann, L., Soderblom, D.R., Jones, B.F., Werner,

M.W., \& McCaughren, M.J. 1994, ApJ, 421, 517

Reipurth, B. \& Zinnecker, H. 1993, A\&A, 278, 81

Shatsky, N. \& Tokovinin, A. 2002, A\&A, 382, 92

Sterzik, M.F. \& Durisen, R.H. 1995, A\&A, 304, 9

Tokovinin, A., Chalabaev, A., Shatsky, N., \& Beuzit, J.L. 1999, A\&A, 346, 481 